

\title{
Imaging Dose in Radiation Therapy
}

Jonathan Sykes, Parham Alaei, and Emiliano Spezi

22.1 Introduction

22.2 Dose Measurement for CT

22.3 Dose Measurement for CBCT for Radiotherapy Applications

22.4 Measurement (and Calculation) of Dose for Planar kV Imaging

22.5 Dose Measurement for MV Portal Imaging

22.6 Dose Measurement for MVCT and MV-CBCT
22.7 Dosimeters for All Modalities

22.8 Dose Calculation Methods

22.9 Estimating Effective Dose and Risk

22.10 Combining Dose from RT and Imaging

22.11 Clinical Consequences and Benefits

22.12 Closing Remarks

\subsection{Introduction}

Previous chapters in this book have concentrated on the instrumentation and measurement techniques for dosimetry of the therapeutic beams. In this chapter, a variety of measurement and calculation techniques will be reviewed for characterizing the radiation dose from x-ray imaging systems used in radiation therapy (RT). X-ray imaging systems are now used extensively throughout a patient's treatment for all complex RTs, and in many cases for simple palliative RTs as well. Nearly, all patients will undergo a multislice computed tomography (CT) examination for localizing the target volume and nearby organs at risk. In addition, a variety of $\mathrm{x}$-ray imaging systems are available to image the patient at the point of treatment, either immediately prior to beam deliver $\bigcirc$ g., Korreman et al., 2010; Moore et al., 2014) to ensure accurate patient angnment, or during beam delivery to monitor intrafraction motion 
(e.g., Ng et al., 2012). The electronic portal imaging device is probably the most basic of these systems and can be used to locate and track anatomy with little or no additional radiation dose to the patient. However, poor image quality limits the device to applications where bony anatomy or radiographic markers are sufficient surrogates for the target anatomy. Megavoltage cone beam $\mathrm{CT}^{*}(\mathrm{MV}-\mathrm{CBCT})$ can be used to provide 3D visualization of soft tissues with little additional hardware but at the cost of increased imaging dose. The addition of a kilovoltage $(\mathrm{kV}) \mathrm{x}$-ray system to the gantry has become the mainstay of $\mathrm{x}$-ray image guidance, providing $\mathrm{CBCT}$ with superior image quality compared to MV-CBCT and the option of planar radiographic or fluoroscopic imaging (Jaffray et al., 1999; Jaffray and Siewerdsen, 2000). Other image guidance systems that utilize x-ray imaging include: Accuray CyberKnife $^{\bullet}$ and the Brainlab ExacTrac system, both of which utilize dual/ stereoscopic kV x-ray imaging systems; Accuray TomoTherapy ${ }^{\oplus}$, which uses helical megavoltage CT (MVCT); and the Mitsubishi VERO radiotherapy unit, which has a dual $\mathrm{x}$-ray imaging system mounted on a rotating gantry capable of stereoscopic $\mathrm{kV}$ imaging, as well as CBCT. These systems have been discussed in detail in Chapter 10.

While all these systems can be used to perform image guidance and enable greater accuracy in the delivery of RT, they all lead to additional x-ray dose to the patient. In imaging intensive CBCT-based image-guided RT (IGRT) regimes this dose can be of the order of Gy for a single CBCT (Spezi et al., 2012). It is therefore important to quantry this dose in order to justify the risks of using $\mathrm{x}$-ray imaging against the benefits for a particular IGRT protocol.

In this chapter, the measurement of imaging dose is reviewed for the various imaging modalities with particular emphasis on $\mathrm{kV}-\mathrm{CBCT}$ imaging.

\subsection{Dose Measurement for CT}

The established and standardized method for measuring fan beam CT dose, since 1981, has been to measure the computed tomography dose index (CTDI) (Shope et al., 1981). CTDI is measured using a 100-mm-long pencil ionization chamber in a cylindrical phantom. The phantom is made of polymethylmethacrylate (PMMA) and is either $32 \mathrm{~cm}$ diameter and $15 \mathrm{~cm}$ length for measurement of dose in the body or $16 \mathrm{~cm}$ diameter and $15 \mathrm{~cm}$ length for measuring dose in the head (Figure 22.1). The CTDI ${ }_{100}$, defined in Equation 22.1, was designed to measure the dose to air over a volume which encapsulates the slice width plus the tails of the fan beam profile on either side. The measurement is performed for a single axial (i.e., not helical) rotation of the x-ray tube without table shift. For a narrow slice $(\leq 1 \mathrm{~cm})$, a $100 \mathrm{~mm}$ long chamber is sufficient to capture enough of the profile without significant loss of accuracy.

$$
\mathrm{CTDI}_{100}=\frac{1}{n T} \int_{-50 \mathrm{~mm}}^{50 \mathrm{~mm}} D(z) \mathrm{d} z
$$

where $n$ is the number of detector rows and $T$ is the thickness of each row (mm).

* MV-CBCT was developed and commercialized by Siemens Medical Systems, but is no longer commercially available since Siemens stopped producing radiation therapy treatment machines. 


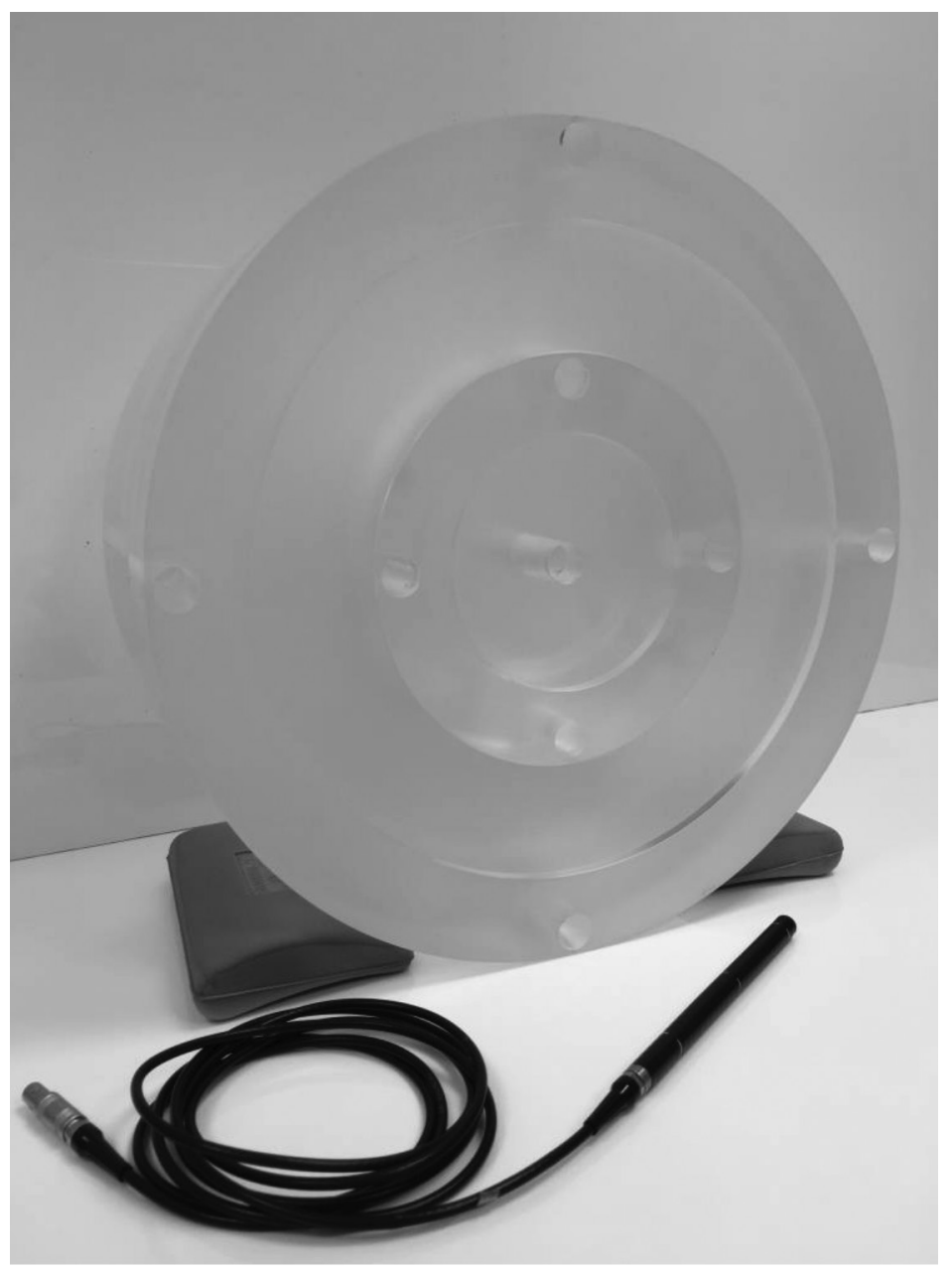

Figure 22.1

Computed tomography dose index (CTDI) phantom with $100 \mathrm{~mm}$ long pencil chamber.

Capturing the entire profile, including the tails due to scatter, is equivalent to measuring the dose for a spiral acquisition. Although there are some variants of the CTDI measurement, the CTDI ${ }_{100}$ is generally accepted as a standard (Kim et al., 2011) and is the standard adopted by the International Electrotechnical Commission (IEC) (2009).

To estimate the average dose in the axial plane, $\mathrm{CTDI}_{w}$, defined in Equation 22.2, is calculated from the average of measurements at the center and the periphery of the phantom.

$$
\mathrm{CTDI}_{w}=\frac{1}{3} \mathrm{CTDI}_{\text {center }}+\frac{2}{3} \mathrm{CTDI}_{\text {periphery }}
$$

where CTDI $I_{\text {center }}$ is the CTDI measured at the center of the phantom and $\mathrm{CTDI}_{\text {periphery }}$ is the average of the dose measured in at least four peripheral points 
on the CTDI phantom. CTDI ${ }_{w}$ alone tells us what average dose in a plane would be given by a series of contiguous axial scans (stepped table feed) or helical scans with a pitch of unity. For a helical scan with pitch not equal to unity, CTDI ${ }_{\text {vol }}$ can be calculated using Equation 22.3. The CTDI ${ }_{\mathrm{vol}}$, when multiplied by the scan length gives the dose length product (DLP), defined in Equation 22.4. If CTDI ${ }_{\mathrm{vol}}$ is expressed in $\mathrm{mGy}$, DLP is generally given in $\mathrm{mGy} \cdot \mathrm{mm}$. It is an estimate of the total absorbed dose in the scanned volume, which can then be related to the stochastic effects of radiation exposure and used as a first-order estimate in public health monitoring (Smith-Bindman et al., 2009).

$$
\mathrm{CTDI}_{\mathrm{vol}}=\frac{\mathrm{CTDI}_{\mathrm{center}}}{\text { Pitch }}
$$

where pitch is the table increment per revolution as a fraction of the detector width $(n T)$, and

$$
\mathrm{DLP}=\mathrm{CTDI}_{\mathrm{vol}} \times L
$$

where $L$ is the scan length.

The introduction of multislice detectors with typical detector widths up to $4 \mathrm{~cm}$ and with some up to $16 \mathrm{~cm}$ has raised discussion about the validity of CTDI (Dixon, 2003; Mori et al., 2005; Boone, 2007). Boone showed that the loss in collection efficiency using a $100 \mathrm{~mm}$ chamber with wider beam widths up to $4 \mathrm{~cm}$ was only a few percent (Boone, 2007). However, it was also shown that the collection efficiency of the $100 \mathrm{~mm}$ chamber for a $1 \mathrm{~cm}$ beam width was only $82 \%$ at the center of the CTDI head phantom. In other words, while CTDI $\mathrm{I}_{100}$ may be a useful dose index, it is not an accurate measure of the equilibrium dose at the center of a long scan. The equilibrium dose is the dose that would be delivered for a long scan of length $L$, where $L$ is considerably longer than $100 \mathrm{~mm}$.

\subsection{Dose Measurement for $\mathrm{CBCT}$ for Radiotherapy Applications}

\subsubsection{CTDI for CBCT}

The CTDI concept starts to break down with increasing cone angle for a number of reasons:

1. The $100 \mathrm{~mm}$ chamber length is not long enough to cover the entire beam profile which is typically comparable to or longer than the beam width in radiotherapy applications.

AU: Please confirm if the edits made to the sentence

"These problems were largely ignored. convey $\mathrm{t}$ intended meaning.

2. The phantom size is not sufficient to capture the entire beam width and its scatter.

3. The weightings of central and peripheral dose in the calculation of $\mathrm{CTDI}_{\mathrm{w}}$ are not necessarily a good estimate of the average dose across the axial plane given the radiological shape of the bow-tie filters used by CBCT systems, and the partial arc scanning utilized by some protocols.

These problems were largely ignored in the studies published soon after the introduction of CBCT in radiotherapy practice (Sykes et al., 2005; Amer et al., 2007; Walter et al., 2007; Osei et al., 2009; Sawyer et al., 2009; Hyer and 
Hintenlang, 2010; Falco et al., 2011), and researchers employed "CTDI-like" techniques to measure the CBCT dose. They continued to use a $100 \mathrm{~mm}$ chamber in a cylindrical PMMA phantom with the same diameters as the body and head CTDI phantoms, 32 and $16 \mathrm{~cm}$, respectively, but lengthened. This method is still used by Varian Medical Systems for measuring CBCT dose for the OBI system (Varian Medical Systems, 2012). To account for the beam length being similar in length or longer than the standard $15 \mathrm{~cm}$, CTDI phantom dose measurements have also been reported with additional scatter material (Amer et al., 2007). In some cases, this has been achieved using two or three CTDI phantoms placed end to end (Palm et al., 2010). In recognition that the $\mathrm{CTDI}_{100}$ only measures an average of the central $100 \mathrm{~mm}$ portion of the CT, some authors introduced the term cone beam dose index (CBDI) (Amer et al., 2007; Osei et al., 2009; Hyer and Hintenlang, 2010).

One method to ensure that the entire dose profile is acquired, at least for cone angles typical of diagnostic CT scanners, is to measure the dose at the center of a lengthened CTDI-type phantom using a $300 \mathrm{~mm}$ long cylindrical ion chamber (Geleijns et al., 2009; Hu and Mclean, 2014) or radiochromic film (Hu and Mclean, 2014).

An alternative to measuring a CTDI-like quantity is to measure the point dose using a $0.6 \mathrm{~cm}^{3}$ Farmer-type chamber, as suggested by Fahrig et al. (2006), in cylindrical PMMA phantoms (Islam et al., 2006; Song et al., 2008; Sykes et al., 2010). Song and collaborators measured the dose at the center of two CTDI phantoms placed end to end using a Farmer-type chamber, and termed this measurement CBCTDI (Song et al., 2008). Sykes and colleagues used the same technique to measure the dose for both the Elekta XVI and Varian OBI systems (Sykes et al., 2010). Typical doses for the two systems, taken from Sykes et al. (2010), are given in Table 22.1.

The equivalence of measuring $D_{L}(z=0)$, the dose at the center of a helical scan of length $L$ and $\mathrm{CTDI}_{L}$, the integral dose for a single static slice between $-L / 2$ and $L / 2$, was demonstrated by Dixon (2003). Furthermore, the equivalence of $D_{L}$ $(z=0)$ was measured at the center of a helical scan (scan length $=L$ and pitch $=1$ ) and a CBCT scan (aperture $a$ ), where $a=L$. This was confirmed experimentally by Mori and colleagues who used a photodiode stepped through the beam to measure profiles of various beam widths (Mori et al., 2005). They showed that dose profiles acquired along the central axis of both helical CT and CBCT scans were equivalent. An alternative to the stepping diode, which would require many

Table 22.1 Cone Beam Dose Measurements (Similar to CTDI ${ }_{w}$ ) for Standard Imaging Protocols on the Varian OBI and Elekta Synergy CBCT Systems

\begin{tabular}{llcllr}
\hline $\begin{array}{l}\text { Varian OBI Imaging } \\
\text { Protocols }\end{array}$ & $\begin{array}{l}\text { Exposure Parameters } \\
(\mathrm{kV} / \mathrm{mAs} / \mathrm{bt})\end{array}$ & $\begin{array}{c}\text { Doses } \\
(\mathrm{mGy})\end{array}$ & $\begin{array}{l}\text { Elekta Synergy } \\
\text { Imaging Protocols }\end{array}$ & $\begin{array}{l}\text { Exposure Parameters } \\
\text { (kV/mAs/bt) }\end{array}$ & $\begin{array}{r}\text { Dose } \\
(\mathrm{mGy})\end{array}$ \\
\hline Low dose head & $100 / 72 / \mathrm{bt}$ & 2.8 & Low dose head & $100 / 36$ & 1.4 \\
Standard dose head & $100 / 145 / \mathrm{bt}$ & 5.6 & Medium dose head & $100 / 144$ & 5.4 \\
High-quality head & $100 / 720 / \mathrm{bt}$ & 27.8 & High dose head & $100 / 288$ & 10.7 \\
Pelvis & $125 / 655 / \mathrm{bt}$ & 24.9 & Pelvis M10 & $100 / 819 / \mathrm{bt}$ & 12.7 \\
Pelvis spotlight & $125 / 360 / \mathrm{bt}$ & 20.2 & Pelvis M15 & $100 / 819 / \mathrm{bt}$ & 14.0 \\
& & & Pelvis M20 & $100 / 819 / \mathrm{bt}$ & 15.3 \\
\hline
\end{tabular}

Note: Manufacturer's recommended protocol settings may change over time based on the introduction of new technology or feedback from customers. (bt signifies a bow-tie filter was used.) 


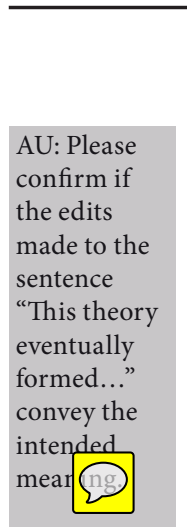

repeat CBCT scans, is the use of a CT dose profiler (RTI Electronics AB, Mölndal, Sweden) as demonstrated by Palm et al. (2010). Based on their respective previous findings, Boone and Dixon proposed a unified and self-consistent approach to both multidetector helical (moving) and stationary CBCT systems by measuring a point dose for a single beam width (Dixon and Boone, 2010). Their theory suggests that this single measurement is sufficient to calculate the dose for a stationary or moving scan of any other beam width. This theory eventually formed the basis of the American Association of Physicists in Medicine (AAPM) Task Group (TG) 111 report on the evaluation of radiation dose in $\mathrm{x}$-ray CT (AAPM, 2010).

\subsubsection{AAPM Recommendations on CBCT Dosimetry}

AAPM TG-111 report (AAPM, 2010) presents the theoretical underpinnings of measuring dose in axial, helical fan beam or CBCT with table translation, as well as in stationary phantom CBCT. For axial or helical scanning, the report notes that there is an equilibrium dose constant which is independent of the collimation or the pitch. The dose for any particular scan can then be determined as the product of the equilibrium dose constant and a factor ( $p n T / a)$ where $p$ is the pitch, $n T$ the total width of the detector, that is, $n$ rows of width $T$, and $a$ is the width of collimation. This considerably reduces the number of measurements that need to be made as long as $a$ is known. The equilibrium dose constant is the dose measured at the central scan plane $(z=0)$ for a scan of length $L_{\text {eq }}$ and with pitch $p=$ a/nT. $L_{\text {eq }}$ has to be sufficiently large to ensure that further increment of $L$ does not significantly increase the measured dose.

For scan lengths shorter than $L_{\mathrm{eq}}$ the dose $D_{L}$, at the center of the scan on the rotation axis, asymptotically approaches $D_{\text {eq }}$ as described by Equations 22.5 and 22.6:

$$
D_{L}(z=0)=h(L) D_{\mathrm{eq}}
$$

and

$$
h(L)=1-\propto \exp \left(\frac{-4 L}{L_{\mathrm{eq}}}\right)
$$

wher 9 eq and $D_{\text {eq }}$ can be determined experimentally by fitting the curve of dose versus the number of scan lengths.

For CBCT, where there is no table movement and typically only one rotation, the dose for a given scan with collimation width $a$ is simply the dose measured at the center of the scan at the midpoint on the central axis. AAPM states that the CBCT dose would also reach an equilibrium for large collimation widths, but $a_{\mathrm{eq}}$ would be greater than $400 \mathrm{~mm}$. Because this is not possible, even on RT image guidance systems, measurement of the dose equilibrium is therefore not clinically relevant. That said, measurement of the dose for a few collimation widths would allow a fit to Equation 22.6, which could then be used for the calculation of the dose for any collimation width used in clinical practice. Since the $100 \mathrm{~mm}$ long chamber does not cover the entire beam profile, it can underestimate the dose by $2 \%-5 \%$ for wide angle CBCT (Osei et al., 2009). This is one reason why the AAPM TG-111 methods are based on measuring the point dose (e.g., using a Farmer-type chamber) in a geometrical phantom (typically cylindrical) that is sufficiently long to provide full scatter conditions for the irradiated scan length. 
AAPM TG-111 also introduces the concepts of integral dose $\left(E_{\text {tot }}\right)$ and planar average equilibrium dose $\left(\overline{D_{\mathrm{eq}}}\right)$, and shows that $E_{\mathrm{tot}}=\rho \pi R^{2} L \overline{D_{\mathrm{eq}}}$ where $R$ is the radius of the volume, $L$ is the scan length, and $\rho$ is the mass density of the phantom. According to this report, the integral dose serves as a simplified indicator of patient risk: the presumption is that cancer risk increases the larger the dose and irradiation volume containing radiosensitive tissue.

The practicalities of measuring $\overline{D_{\mathrm{eq}}}$, the average dose over the scan plane are discussed briefly. Recognizing that when $D_{\text {eq }}(r)=A+B r^{2}$, that is, $D_{\text {eq }}(r)$ has a parabolic form, the measurement of $D_{\text {eq }}(r)$ at two points, such as in the center and in the periphery of the phantom as conventionally measured for CTDI $_{\mathrm{vol}}$, would be reasonable. Note, however, that this leads to $E_{\text {tot }}=1 / 2 \cdot D(r=0)+1 / 2 \cdot D(r=R-1)$ instead of more commonly used formula $\mathrm{CTDI}_{\mathrm{vol}}=1 / 3 \cdot \mathrm{CTDI}(r=0)+2 / 3 \cdot \mathrm{CTDI}(r=R-1)$. Recognizing that $D_{\text {eq }}(r)$ does not always follow a parabolic form, AAPM TG-111 notes that more detailed measurements or the use of Monte Carlo (MC) modeling might be required. This would be the case for the large fields of view of the Elekta XVI and Varian OBI CBCT systems, and for the medium field of view of the Elekta system where the detector panel is shifted laterally to extend the field of view. This creates a central cylinder which is exposed from all $360^{\circ}$ while material in the remaining volume is only exposed from $180^{\circ}$. In addition, the very different bow-tie filter designs between the Elekta and Varian systems will lead to different radial dose profiles.

AAPM TG-111 notes that measuring the free-in-air dose equilibrium pitch product $^{*}$ is an important measurement to make at commissioning as it can be used for quality assurance purposes to assess constancy of exposure and to infer the equilibrium dose measured in a phantom given a scanner with the same phantom factor (ratio of dose equilibrium in phantom to free-in-air dose equilibrium).

While AAPM TG-111 presents the theoretical underpinnings of the measurement of CBCT dose, it does not offer much in the way of standardization of $\mathrm{CBCT}$ dose measurement. The report suggests, but does not dictate, the use of a Farmer-type chamber. It also discusses various phantom designs with different dimensions and cross-sectional shapes (circle or ellipse) and different materials, but does not make any recommendations for a standard phantom and chamber as with the CTDI concept. The methods used by Islam et al. (2006), Song et al. (2008), and Sykes et al. (2010) are in many ways closely aligned with those of AAPM TG-111.

\subsubsection{IAEA Recommendations for CBCT Dosimetry}

The IAEA has published an update on the status of CT dosimetry for wide-cone beam scanners (IAEA, 2011) which includes recommendations on CT and CBCT dosimetry based on the IEC 60601-2-44 report (IEC, 2009). This is a more pragmatic approach to dosimetry of CBCT scanners than the AAPM recommendations and can be performed with current dosimetry equipment. They noted that even for a $10 \mathrm{~mm}$ wide beam $\mathrm{CTDI}_{100}$, measured at the center of the phantom, only $82 \%$ and $63 \%$ of the dose is collected for the head and body phantoms, respectively (Boone, 2007). This illustrates that the $\mathrm{CTDI}_{100}$ was never as accurate

\footnotetext{
* The product of the pitch with the free-in-air dose equilibrium corrects for variation in dose due to choice of pitch.
} 
as one might desire with doses underestimated for long scan lengths and overestimated for short scan lengths. However, the CTDI ${ }_{100}$ accuracy stayed constant for beam widths between 10 and $40 \mathrm{~mm}$, and only decreased significantly for beam widths greater than $40 \mathrm{~mm}$. The IAEA recommends a two-tier approach to the CTDI ${ }_{100}$ with measurements for beam widths of less than $40 \mathrm{~mm}$ following the existing method, but for those greater than $40 \mathrm{~mm}$ they exploit Equation 22.7 which states that the CTDI for a beam width greater than $40 \mathrm{~mm}$ is related to the CTDI for a beam width less than $40 \mathrm{~mm}$ by the ratio of the CTDI ${ }_{\text {free-in-air }}$ at the two beam widths.

$$
\mathrm{CTDI}_{100,(N \times T)>40}=\mathrm{CTDI}_{100, \text { ref }} \times\left(\frac{\mathrm{DTDI}_{\text {free-in-air }, N \times T}}{\mathrm{CTDI}_{\text {free-in-air,ref }}}\right)
$$

where $\mathrm{CTDI}_{100, \text { ref }}$ is the $\mathrm{CTDI}_{100}$ measured in a phantom for the reference beam of $(N \times T)_{\text {ref }}$ using an integration of $100 \mathrm{~mm}, N$ is the number of detector rows, $T$ is the thickness of a single detector row and where $(N \times T)_{\text {ref }}$ is typically $20 \mathrm{~mm}, \mathrm{DTDI}_{\text {free-in-air }, N \times T}$ is the $\mathrm{CTDI}_{\text {free-in-air,ref }}$ for a beam width of $N \times T$, and $\mathrm{CTDI}_{\text {free-in-air,ref }}$ is the $\mathrm{CTDI}_{\text {free-in-air }}$ for the reference beam width.

The measurement of $\mathrm{CTDI}_{\text {free-in-air, ref }}$ is itself done in two tiers with a single chamber (100 mm length) position used for beam widths less than $60 \mathrm{~mm}$; and two or three positions, each stepped by $100 \mathrm{~mm}$ to cover beam widths larger than $60 \mathrm{~mm}$. Note that the IAEA formalism results in a dose index equivalent to the CTDI ${ }_{100}$ but for a wider beam width, and therefore, retains the fundamental problem that the collection efficiency of the $100 \mathrm{~mm}$ pencil chamber is considerably less than $100 \%$ as described previously. The IAEA formulation is not equivalent to measuring the dose that would be given for a particular scan using, for example, a Farmer-type chamber at the center of a large phantom or using a $300 \mathrm{~mm}$ pencil chamber $\left(\mathrm{CTDI}_{300}\right)$ (Hu and Mclean, 2014).

\subsubsection{Comparison of AAPM and IAEA Results}

$\mathrm{Hu}$ and McLean measured CBCT dose using both the AAPM and IAEA methods of CT dosimetry and compared the results with those obtained with the previous $\mathrm{CTDI}_{\mathrm{w}, 100}$ method as used by Varian to measure CBCT dose for the OBI system (Hu and Mclean, 2014). They used three CTDI phantoms stacked end to end, and a variety of dosimeters including a PTW $30009100 \mathrm{~mm}$ pencil chamber, a PTW $30017300 \mathrm{~mm}$ pencil chamber, an IBA Farmer-type chamber, and Gafchromic XR QA2 film. Taking a standard head protocol with a $184 \mathrm{~mm}$ beam width as an example they found that $\mathrm{CTDI}_{\mathrm{w}, 100}$ was $5.53 \mathrm{mGy}$ in a $15 \mathrm{~cm}$ phantom with a $100 \mathrm{~mm}$ pencil chamber and $4.28 \mathrm{mGy}$ using the IAEA correction factor. In a 45 $\mathrm{cm}$ phantom, $\mathrm{CTDI}_{\mathrm{w}, 300}$ was $5.48 \mathrm{mGy}$ with a $300 \mathrm{~mm}$ pencil chamber. Using the AAPM formalism with a $0.6 \mathrm{~cm}^{3}$ Farmer-type chamber, the dose was $5.49 \mathrm{mGy}$. For a pelvis scan with a $206 \mathrm{~mm}$ beam width, the $\mathrm{CTDI}_{\mathrm{w}, 100}$ in a $15 \mathrm{~cm}$ phantom was 18.06 and $15.88 \mathrm{mGy}$ with and without the IAEA correction, respectively, and in the $45 \mathrm{~cm}$ phantom $\mathrm{CTDI}_{\mathrm{w}, 300}$ was $22.37 \mathrm{mGy}$. The AAPM formalism gave $22.7 \mathrm{mGy}$.

As Hu and McLean comment, there is good agreement between the CTDI $\mathrm{w}_{3,300}$ and the Farmer-type $0.6 \mathrm{~cm}^{3}$ chamber measurements, despite one measuring the combined central and peripheral integrated dose profiles, and the other the peak central dose. As expected, they showed a clear difference between $\mathrm{CTDI}_{\mathrm{w}, 300}$ and $\mathrm{CTDI}_{\mathrm{w}, 100}$ measured in 45 and $15 \mathrm{~cm}$ phantoms due to the loss of collection 
efficiency of the $100 \mathrm{~mm}$ chamber and the lack of scatter material. No comment was made in the article about the IAEA corrected CTDI ${ }_{w, 100}$ measurement, which seems to reduce the dose further from what could be considered the gold standard using the $\mathrm{CTDI}_{\mathrm{w}, 300}$ measurement.

\subsection{Measurement (and Calculation) of Dose for Planar kV Imaging}

In radiation oncology, $\mathrm{kV}$ x-ray imaging systems are used for IGRT in both radiographic and fluoroscopic modes (Yin et al., 2009). Radiographic modes are typically used to ensure correct placement of the isocenter and patient alignment using two orthogonal views, usually anterior-posterior and lateral. The use of implanted radiographic markers can make this a highly accurate method of aligning the target volume (Schiffner et al., 2007). Fluoroscopic modes can be used to monitor respiration prior to treatment, for example, to monitor diaphragm position, to ensure correct positioning for deep inspiration breath hold (DIBH) techniques in treatment of breast cancer (Borst et al., 2010), or to monitor target motion during treatment at sites such as prostate, lung, liver, and pancreas (Shirato et al., 2004; Ng et al., 2012). In this section, both radiographic and fluoroscopic modes will be considered equivalent for the purpose of measuring the radiation dose. The difference between the two is simply that in radiographic mode the $\mathrm{x}$-ray tube is switched on once, while in fluoroscopic mode the tube is pulsed once per image frame acquired and can therefore be considered as a sequence of radiographic images.

In diagnostic radiology, there are two primary concerns: (1) the deterministic effects of radiation exposure, for example, skin erythema from long exposures where the skin dose exceeds $2000 \mathrm{mGy}$, and (2) the stochastic risk of secondary induced cancer from radiation exposure. The first effect can be determined from a measurement using an ion chamber, thermoluminescent dosimeters (TLDs), or optically stimulated luminescent dosimeters (OSLDs). The second effect can be estimated from the effective dose. AAPM TG-75 report (Murphy et al., 2007) provides an example of the estimation of effective dose for a particular radiographic exposure. This example uses the entrance dose given as dose area product (DAP) multiplied by a factor F, which is specific to a technique and is derived using MC calculations of dose in a mathematical phantom. The MC technique is described in NRPB Report 186 (Jones and Wall, $1985)$ and the $F$-factors for a variety of radiographic procedures have been calculated by Le Heron (1992).

It is not standard practice to characterize the 3D dose distribution in a homogeneous phantom in diagnostic radiology. Measuring the DAP is sufficient in the diagnostic community to estimate the effective dose for a procedure as described above.

In $\mathrm{RT}$, there are two purposes of measuring the radiation dose from radiographic/fluoroscopic procedures: (1) to calculate effective dose ${ }^{\star}$ from the imaging to be combined with the effective dose from the treatment beam/source as recommended by AAPM TG-75 (Murphy et al., 2007), and (2) to calculate the

\footnotetext{
* Effective dose, as defined by Jacobi (1975) is "the mean absorbed dose from a uniform whole-body irradiation that results in the same total radiation detriment as from the non-uniform, partialbody irradiation in question" (Equation 22.10).
} 
additional dose to critical organs which may already be receiving a threshold dose from the treatment beam/source.

For the calculation of effective dose, it is not necessary to calculate dose for

AU: Please confirm if the edits made to the sentence "The calcula tion of do to..." contey the intended meaning. an individual patient and therefore use of effective dose calculations based on the entrance dose are deemed to be sufficient. The calculation of dose to individual organs ideally should be done based on the individual patient anatomy using either MC methods or other algorithms as proposed by several groups (Ding et al., 2008b; Spezi et al., 2009; Alaei et al., 2010; Pawlowski and Ding, 2014; Poirier et al., 2014) discussed in Section 22.8. Unfortunately, these techniques are not widely available. In the absence of such methods, it may be sufficient to estimate dose to organs based on knowledge of the basic depth dose distribution of $\mathrm{kV} \mathrm{x}$-rays in water. In order to measure the dose distribution of $\mathrm{kV} x$-rays, the RT medical physicists can turn to techniques for characterizing the dose in superficial x-ray units. For the absolute dose calibration, there are a number of protocols to choose from, for example, the Institution of Physics and Engineering in Medicine and Biology (IPEMB) code of practice (IPEMB, 1996), the IAEA TRS-398 code of practice (IAEA, 2000), and AAPM Report 61 (Ma et al., 2001). Methods of relative dosimetry for $\mathrm{kV}$ x-ray beams have been reviewed comprehensively by Hill et al. (2014) in the context of superficial x-ray treatment systems, and include details of measurement using ion chambers, diamond detectors, diodes, metal-oxide-semiconductor field-effect transistors (MOSFETs), optical fibers, OSLDs, plastic scintillator detectors, TLDs, radiochromic film, and gel dosimeters.

Typical doses for radiographic and fluoroscopic systems vary widely depending on the technique and the anatomical site being imaged. In particular, fluoroscopic imaging doses will depend on the length of the procedure and the pulse-repetition frequency. Tien and colleagues compared the entrance skin exposure (ESE) for two centers treating brain, thorax, abdomen, and pelvic regions with the CyberKnife system. They found the average ESE to be 17, 53, 41, and 68 cGy, respectively, for these regions (Tien et al., 2014). In a white paper published by Accuray (Accuray, n.d.), effective doses of 0.24, 3.56, and $16 \mathrm{mSv}$ were reported for image guidance with intrafraction tracking of motion for the head, chest, and pelvis with a total number of projection images of 54, 138, and 196, respectively.

Ding and Munro (2013) compared radiation dose values from an orthogonal pair of MV portal images, $\mathrm{kV}$ radiographs, and CBCT in the head, thorax, and pelvic regions. They performed MC simulations to determine the portal imaging doses and concluded that $\mathrm{kV}$ radiographs deliver the least dose among imaging modalities with typical doses in the order of a fraction of a cGy.

\subsection{Dose Measurement for MV Portal Imaging}

Portal imaging using the MV photon beam and an image receptor, for example, radiographic film, which was subsequently replaced by electronic portal imaging devices, was the principle imaging tool prior to development of CBCT, and is still being utilized heavily for patient position verification. This is often accomplished by taking AP and lateral radiographs to verify isocenter location, but may involve imaging individual beam portals, in which case the images are often double exposed by imaging both with the beam-limiting devices (blocks, multileaf collimators [MLCs]) in place and without them. 
The portal imaging dose has traditionally been estimated by equating each monitor unit (MU) delivered for an image to one cGy, which is obviously not accurate as the patient dose varies depending on the imaging field size and patient size. Jones and Shrimpton (1991) measured the portal film dose for 100 patients and reported average doses of up to $150 \mathrm{cGy}$ per course of linac-based treatment. More recently, Kudchadker et al. (2004) evaluated radiation exposure from portal films in pediatric patients and reported mean total doses per course of RT to be between 17 and $46 \mathrm{cGy}$, with most of the dose due to open-field dose from the double-exposure technique. Additional in vivo studies provide further data for dose using electronic portal imaging systems both at the surface and internally. Walter et al. (2007) measured the electronic portal imaging dose to patients and reported a skin dose of 5.8-6.9 cGy and a rectal dose of approximately $3 \mathrm{cGy}$ for a pair of portal images. Stock et al. (2012) measured the electronic portal imaging dose in an anthropomorphic phantom and reported doses ranging between 3 and 5 cGy. Ding and Munro (2013) reported MC calculated doses for MV electronic portal imaging to be in the order of 2-4 cGy per orthogonal pair.

The reduction of MV portal imaging dose is achievable by: (1) limiting the collimator size in double-exposure imaging and (2) using fewer MUs in imaging smaller volumes, such as in the head and neck region and for pediatric cases.

\subsection{Dose Measurement for MVCT and MV-CBCT}

MVCT scanning in RT is exclusive to TomoTherapy systems (Accuray, Sunnyvale, CA). The TomoTherapy Hi Art system utilizes a 3.5 MV x-ray beam and a row of xenon detectors to acquire fan beam CT images for patient position adjustments. During imaging, a $4 \mathrm{~mm}$ jaw width (as projected to isocenter) is used for all image acquisitions. The MVCT images are acquired at couch speeds of 4,8 , and $12 \mathrm{~mm} /$ rotation, corresponding to pitch values of 1,2 , and 3, referred to as fine, normal, and coarse image acquisition, respectively. The images are then reconstructed as fine, normal, and coarse, corresponding to slice thicknesses of 1 or 2, 2 or 4 , and 3 or $6 \mathrm{~mm}$, respectively, the thinner of each set is obtained from interpolation. Thus, the acquisition pitch determines the slice thickness, imaging dose, and duration of image acquisition (Shah et al., 2008). The imaging dose is also dependent on the length of imaged volume and patient size. The reported measured doses range from less than $1 \mathrm{cGy}$ to over $2 \mathrm{cGy}$ in cylindrical and anthropomorphic phantoms (Shah et al., 2008) utilizing multiple scan average dose (MSAD) measurements. Similar types of measurements in a cylindrical acrylic phantom reported doses between 0.2 and $1 \mathrm{cGy}$ for pitches between 4 and 1 (Meeks et al., 2005). Another set of measurements using TLDs in an anthropomorphic phantom indicated imaging doses of $<1 \mathrm{cGy}$ for coarse setting (Shah et al., 2012).

Imaging dose from MV-CBCT employed in Siemens linacs has been measured by several groups (Gayou et al., 2007; Morin et al., 2007a,b; Isambert et al., 2009; Quinn et al., 2011; Halg et al., 2012). The dose from this imaging modality generally increases with higher MU protocol, which produces better quality images. Due to fixed gantry start/stop angles, there is also a steep dose gradient within the patient with higher dose on the anterior portions of the body, assuming supine position. This has been illustrated by Miften and colleagues using a treatment-planning system (Miften et al., 2007). The reported doses from $\mathrm{MV}$-CBCT imaging range from a fraction of cGy up to $12 \mathrm{cGy}$, depending on the protocol used. 


\subsection{Dosimeters for All Modalities}

Virtually any dosimeter used in RT can be used for imaging dose measurements provided it has been calibrated for the quality of the imaging beam and characterized for its behavior in such a beam quality. Ion chambers can be used to measure dose from any imaging beam, regardless of beam quality, with the caveat that they need to be calibrated for the imaging beam quality if different than the therapeutic one. For example, to use an ion chamber in the $\mathrm{kV}$ energy range, the chamber must be calibrated for that beam quality with traceability to a dosimetry standard laboratory.

TLDs have been extensively used for dose measurements in anthropomorphic

AU: "Dufek et al., 2011" is not listed in the Reference list. Please provide complete reference details or remove text citation. phantoms and kV-CBCT beams (Sykes et al., 2005; Amer et al., 2007; Saw et al., 2007; Wen et al., 2007; Kan et al., 2008; Marinello et al., 2009; Osei et al., 2009; Palm et al., 2010; Cheng et al., 2011; Dufek et al., 2011; Halg et al., 2012). They have also been used for skin dose measurements from imaging beams. One of the limitations of TLDs is the energy dependence of their response; hence, to use them for measurements in $\mathrm{kV}$ beams they either have to be calibrated for the same beam quality or their response corrected using a correction factor (Kron et al., 1998; Nunn et al., 2008).

Among other dosimeters commonly available, OSLDs can also be used for imaging dose measurements. The use of OSLDs in $\mathrm{kV}$ x-ray beams has been studied by several groups (Winey et al., 2009; Ding and Malcolm, 2013; Giaddui et al., 2013).

Other dosimeters such as MOSFETs (Cheung et al., 2003; Ehringfeld et al., 2005), radiographic, and radiochromic films have also been used for MV imaging dose measurements and could be used for $\mathrm{kV}$ measurements as well (Marinello et al., 2009; Isambert et al., 2009; Alvarado et al., 2013; Giaddui et al., 2013; Nobah et al., 2014).

\subsection{Dose Calculation Methods}

\subsubsection{Dose Calculation Algorithms for MV-CBCT}

In case of MV-CBCT, all the current algorithms available in treatment-planning systems can be utilized to compute the imaging dose as the same $6 \mathrm{MV}$ therapeutic beam is used for imaging. This has been done by several groups, indicating its feasibility and the ability of including MV-CBCT dose in treatment planning (Miften et al., 2007; Morin et al., 2007a,b). The Siemens units can also perform MV-CBCT using an "Imaging beam line" (IBL), which employs a degraded 4.2 $\mathrm{MeV}$ beam and a carbon target to produce the imaging beam (Faddegon et al., 2008). The IBL beam data has been collected and modeled in one commercial treatment-planning system utilizing a convolution/superposition algorithm (Flynn et al., 2009). Figure 22.2 shows a dose distribution from MV-CBCT using a treatment-planning system.

\subsubsection{Dose Calculation Algorithms for kV-CBCT}

Calculating $\mathrm{kV}$-imaging dose using available algorithms poses greater challenges as these algorithms have been developed to calculate the dose from MV beams which predominantly interact with tissue through Compton interactions. Interactions in the $\mathrm{kV}$ range are predominantly through photoelectric effect, which is not modeled accurately with these algorithms, with the exception of 




Figure 22.2

Distribution of dose deposited in the pelvis by a single fraction of MV-CBCT imaging for a prostate patient, with $10 \mathrm{cGy}$ at isocenter. The isodose lines are labeled in CGy. (Reproduced from Miften M et al., Med. Phys., 34, 3760-3767, 2007. With permission.)

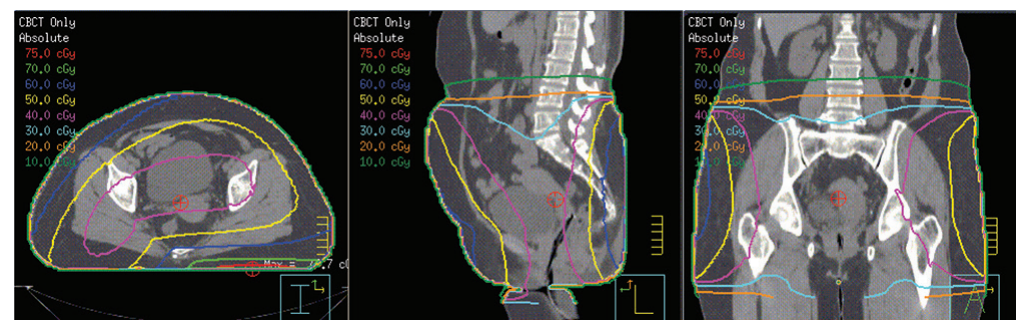

Figure 22.3

Isodose distribution showing the imaging dose from 25 fractions of pelvic imaging for one patient using the Elekta XVI pelvis imaging protocol ( $120 \mathrm{kVp}, 1 \mathrm{mAs}, 650$ projections) calculated using the Pinnacle treatment-planning system. (Reproduced from Alaei P and Spezi E, Phys. Med., 31, 647-658, 2015. With permission.)

MC methods. A common algorithm applied in treatment-planning systems, convolution/superposition, has been used for dose calculation from $\mathrm{kV}$-CBCT by addition of $\mathrm{kV}$ energy deposition kernels (Alaei et al., 2010) producing reasonable results in soft tissue and lung but underestimating the dose in and around bone (Alaei et al., 2001, 2010). Convolution-based algorithms do not account for atomic number changes in the medium, which are needed for accurate dose calculations in the $\mathrm{kV}$ range. A proposed algorithm (Ding et al., 2008b) overcomes this problem by introducing a correction factor to account for atomic number changes. This algorithm is currently not available commercially. Figure 22.3 demonstrates such a dose calculation using the convolution-based algorithm implemented in the Pinnacle treatment-planning system (Philips, Milpitas, CA).

\subsubsection{Dose Calculation Using MC Methods}

Frequently used methods for estimating dose from CBCT are based on calculating the dose to either the CTDI phantoms or to simplified humanoid computational phantoms. In the diagnostic world of radiation protection, it may be sufficient to relate the dose for CT scan protocols used by a particular hospital to the radiation risk for the purpose of justification and for reporting dose. 
However, in radiotherapy where many CBCT scans might be performed during the course of treatment, it may be necessary to calculate the dose to specific critical organs to ensure that the combined treatment and concomitant imaging dose does not exceed the dose criteria specified in the treatment protocol. In such cases, individualized patient dose calculations may be required. The MC method is regarded as the most accurate approach to model ionizing radiation transport for radiotherapy and imaging applications (Verhaegen and Seuntjens, 2003; Spezi and Lewis, 2008), and it is an ideal tool for CBCT patient dosimetry. The calculation of concomitant dose from both $\mathrm{kV}$ - and MV-CBCT units has been carried out extensively with the EGSnrc code system (which includes the BEAMnrc and DOSXYZnrc codes) and, to a lesser extent, with other MC codes such as MCNP and Geant4 (Chow et al., 2008; Ding et al., 2008a; Gu et al., 2008; Ding and Coffey, 2009, 2010; Downes et al., 2009; Spezi et al., 2009, 2011, 2012; Walters et al., 2009; Qiu et al., 2011, 2012; Deng et al., 2012a,b; Zhang et al., 2012; Ding and Munro, 2013; Son et al., 2014). As reported by Alaei and Spezi (2015), several groups developed MC models for CBCT imaging systems and calculated 3D dose distributions using patient-specific CT scans or virtual phantoms. This is the result of several works aimed at improving particle transport models for $\mathrm{x}$-ray photon beams in the diagnostic energy range (Kawrakow, 2013). The commissioning of an MC model for a CBCT unit is in principle similar to the commissioning of a treatment-planning system for external beam radiotherapy. First, the model of the unit's head, including source, filters, and beam collimators, has to be built. Second, the model has to be calibrated for absolute dose calculation, and dose profiles obtained in reference conditions must be validated against experimental measurements. The process for the absolute dose calibration of a CBCT MC model was described by Ding et al. (2008a) and Downes et al. (2009). The MC calibration factor, defined in Equation 22.8, is specific to each CBCT beam and is derived by measuring, in reference conditions, the absolute dose to a point in a phantom with known geometry, and by calculating the $\mathrm{MC}$ dose to the same point.

$$
F_{\mathrm{MCcal}}=\frac{D_{\text {exp }}}{D_{\mathrm{MCcal}}}
$$

where $D_{\exp }$ is the measured dose in units of Gy and $D_{\text {MCcal }}$ is the MC dose, calculated in the same reference conditions, in units of Gy per incident particle.

Once the computational model is commissioned, 3D dose calculation can be carried out by sampling the photons incident on the patient with one of the following methods using: (1) full MC simulation of the beam line (Qiu et al., 2011, 2012); (2) a phase space file representing the invariant parts of the unit or fixed field sizes (Chow et al., 2008; Ding et al., 2008a; Ding and Coffey, 2009, 2010; Downes et al., 2009; Walters et al., 2009; Spezi et al., 2012); (3) a source model representing the main sources of radiation (Spezi et al., 2011; Deng et al., 2012a,b; Zhang et al., 2012; Ding and Munro, 2013; Montanari et al., 2014); and (4) an x-ray spectrum (Gu et al., 2008; Ding et al., 2010). Several groups (Chow et al., 2008; Downes et al., 2009; Spezi et al., 2009, 2011, 2012) have developed a computational model for the Elekta XVI CBCT unit using the EGSnrc/BEAMnrc code system and Beampp (a C++ implementation of the BEAMnrc MC code). A number of other groups have developed MC models of the Varian OBI CBCT scanner (Gu et al., 2008; Qiu et al., 2011, 2012; Zhang et al., 2012; Ding and Munro, 2013; 
Montanari et al., 2014). All publications reported 3D dose data calculated on voxelized geometries representing human anatomy, based on patient CT scans or virtual phantoms, and present doses to various organs of interest. While it is not feasible to list all the organ dose data in this chapter, we summarize in Table 22.2 the typical $\mathrm{kV}$-CBCT doses for three anatomical sites. Note that the performance of the CBCT systems in Table 22.2 should not be judged on the basis of the data reported, since there is no reason to assume that the acquisition settings have been optimized to give the same trade-off between imaging dose and image quality.

The following observations are generally applicable to understanding patient dose from CBCT. As shown in Figure 22.4, MC simulations have demonstrated that bony structures can receive two to four times the dose delivered to the soft tissue (Ding et al., 2008a; Downes et al., 2009; Spezi et al., 2012). Furthermore, the use of computational phantoms based on micro-CT images pointed out that the average dose to bone surface cells can be up to $80 \%$ higher than the average dose to organs at risk in a typical head and neck CBCT scan (Walters et al., 2009). This is caused by the increased mass-energy absorption coefficient due to the photoelectric interaction within the materials of higher atomic number. It has also

Table 22.2 Typical MC Calculated Patient Doses, in cGy, for Three Anatomical Sites for the Elekta Synergy CBCT System and the Varian OBI CBCT System

\begin{tabular}{lccc}
\hline & Pelvis/Abdomen & Head and Neck & Chest \\
\hline Elekta XVI (Spezi et al., 2012) & $1.5-3.3$ & $0.1-0.2$ & $1.2-3.4$ \\
Varian OBI (Ding and Coffey, 2009; & $1-5$ & $0.2-0.5$ & $2-9$ \\
Montanari et al., 2014) & & &
\end{tabular}

Note: Doses reported are for the body, that is, not for a specific organ.

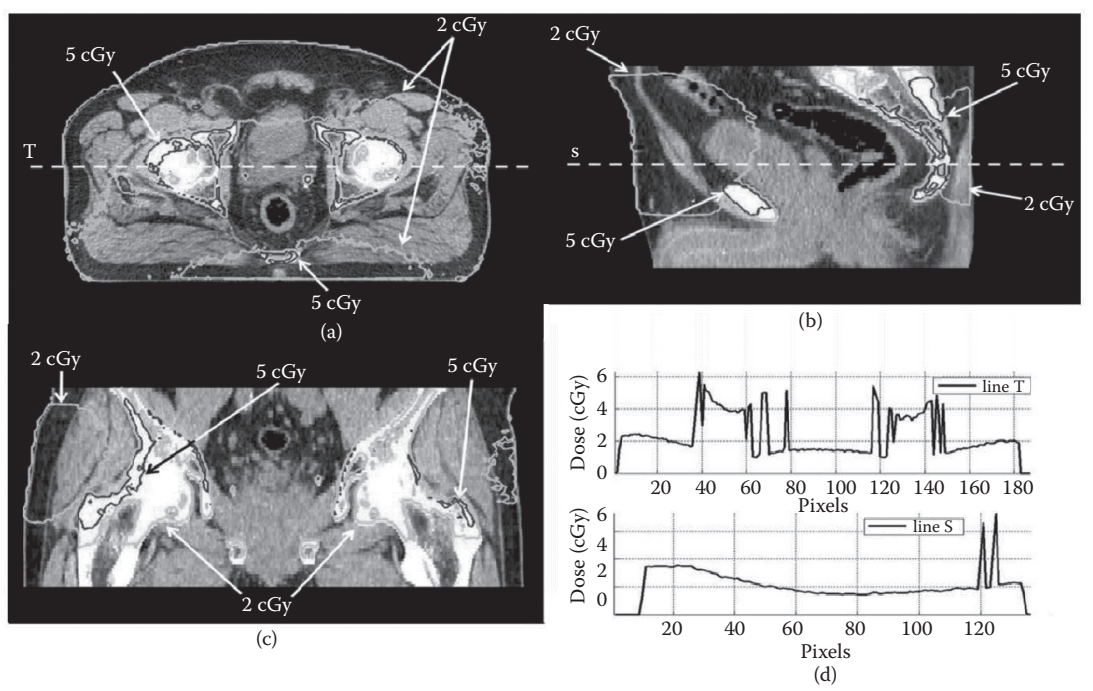

Figure 22.4

Patient dose from XVI CBCT pelvis scan simulated using the M10 collimator and Fl bow-tie filter. (a) Transverse, (b) sagittal, and (c) coronal dose contours are shown. Absolute dose profiles in the transverse and sagittal plane are shown in (d). (Reproduced from Downes P et al., Med. Phys. 36, 4156-4167, 2009. With permission.) 
been shown that the addition of the bow-tie filter significantly reduces the dose by $22 \%$ in the pelvis and $45 \%$ in the chest (Spezi et al., 2012). This is primarily due to the attenuation of the dose to the peripheral tissues but a to the beam hardening effect. Moreover, the bow-tie filter reduces the scattered dose from the periphery of the patient to the imager which has the additional advantage of increasing image quality.

Downes et al. (2009) also showed that the CBCT imaging dose has a left-right asymmetry due to the increased number of exposures at the start and stop gantry angles as the gantry rotation accelerates and decelerates at the beginning and end of each scan. Unlike CT imaging where the patient is normally central in the CT scanner, in radiotherapy the isocenter is typically set to the center of the target volume which may itself be offset from the center of the patient's cross section. Chow (2009) studied this effect and found for the pelvis phantom variation in the mean dose of up to $20 \%$ for up to $10 \mathrm{~cm}$ anterior-posterior shifts. Dose variations for the chest and head and neck were typically between $7 \%$ and $17 \%$. It has been found that $\mathrm{kV}-\mathrm{CBCT}$ doses are highly (inversely) correlated with patient size, expressed in weight or body mass index (BMI) (Zhang et al., 2012; Alaei et al., 2014). In particular, doses to pediatric patients were found to be of the order of two times that of an adult (Ding et al., 2008; Deng et al., 2012a).

\subsection{Estimating Effective Dose and Risk}

The CTDI ${ }_{\mathrm{vol}}$ measurement is an estimate of the average dose in the central axial plane of the scan and is typically calculated as one-third of the central dose and two-thirds of the peripheral dose, as discussed in Sections 22.2 and 22.3. This is independent of the scan length and therefore does not relate to the total dose to the patient and risk of radiation-induced malignancy. A commonly used and very simple metnod to relate $\mathrm{CTDI}_{\mathrm{vol}}$ to total imaging dose is to multiply it by the length of the scan. This is known as the dose length product and has been discussed in Section 22.2. Unlike the above, the quantity referred to as integral dose (total energy absorbed in a volume) can be used as a surrogate to estimate patient risk, assuming that the risk increases with the dose and volume irradiated. AAPM TG-111 (AAPM, 2010) presents the methodology to relate integral dose to scan length and other quantities as elucidated in Section 22.3.2.

For a more accurate assessment of radiation risk, the dose to individual organs and their respective organ sensitivities are needed. The effective dose, defined in Equation 22.10, measured in units of sievert (Sv), is a summation of tissue equivalent doses, shown in Equation 22.9, and tissue-specific weighting factors defined in ICRP Report 103 (ICRP, 2007). The effective dose can be related to radiation risk using, for example, data presented in the Biological Effects of Ionizing Radiation (BEIR) Report published by the National Academies Concerning Radiation Health Risks (BEIR, 2006). The equivalent dose $H_{\mathrm{T}}$ for tissue/organ $\mathrm{T}$ is given by

$$
H_{\mathrm{T}}=\sum_{\mathrm{R}} W_{\mathrm{R}} \cdot D_{\mathrm{T}, \mathrm{R}}
$$

where $W_{\mathrm{R}}$ is the weighting factor for radiation type $\mathrm{R}$ and $D_{\mathrm{T}, \mathrm{R}}$ is the absorbed dose for tissue $\mathrm{T}$ by radiation type $\mathrm{R}$. The effective dose $E$ is then given by

$$
E=\sum_{\mathrm{T}} W_{\mathrm{T}} \cdot H_{\mathrm{T}}
$$


where $W_{\mathrm{T}}$ is the weighting factor iven in ICRP Report 103 (ICRP, 2007) and $H_{\mathrm{T}}$ is the equivalent dose for tissue or organ type $\mathrm{T}$.

One method of measuring organ dose to calculate effective dose is to use an anthropomorphic phantom for CBCT. This has been performed by a number of groups using small radiation dosimeters such as TLDs (Sykes et al., 2005; Amer et al., 2007; Wen et al., 2007; Osei et al., 2009; Sawyer et al., 2009; Palm et al., 2010; Stock et al., 2012), fiber optic-coupled water-equivalent plastic scintillators (Hyer et al., 2010), silicon-photodiode dosimeters (Koyama et al., 2010), and MOSFETs (Perks et al., 2008; Kim et al., 2010).

An alternative method of estimating the effective dose is to use the ImPACT CT patient dose calculator (http://www.impactscan.org/ctdosimetry.htm). The ImPACT dose calculator, designed originally for fan beam CT, uses a library of MC calculated dose calculations (Jones and Shrimpton, 1991) for organ doses in a humanoid mathematical phantom. The library covers numerous commercial CT scanners each characterized by the ratio of peripheral to central CTDI ${ }_{w}$ and central to in-air $\mathrm{CTDI}_{\mathrm{w}}$ for both the head and body phantoms. To calculate the effective dose, the operator selects the scanner type and the start and stop positions of the scan. The software will provide individual organ doses with their weighting factors and equivalent doses, as well as the total effective dose. Ideally when using the ImPACT calculator, the CT scanner for which the dose is to be calculated will be one of the scanners in the ImPACT library. If not, the CT scanner can be matched to the closest one in the library using ImPACT factors derived from a linear combination of the ratios of the central and peripheral normalized CTDI ${ }_{100}$ to CTDI $_{\text {air }}$. This method has been employed by several authors to match a CBCT scanner with fan beam CT scanners in the ImPACT library (Amer et al., 2007; Sawyer et al., 2009). Hyer and Hintenlang (2010) compared organ doses from the ImPACT dose calculator with previously published MC calculated organ doses (Hyer et al., 2010). They found that many organs agreed within 40\%, with generally better agreement for the pelvis scan. However, some discrepancies of more than $100 \%$ were also found. They concluded that the ImPACT dose calculator is not suitable for calculating CBCT dose.

$\mathrm{Gu}$ et al. have modeled both $\mathrm{kV}$ - and MV-CBCT systems using MCPNX (Gu et al., 2008) and applied these models to calculate organ doses to the VIPMan phantom that was developed from the National Library of Medicine's Visible Human Project (Xu et al., 2000). They concluded that the effective dose for the head and neck and prostate was 8.53 and $6.25 \mathrm{mSv}$, respectively, for a $125 \mathrm{kVp} \mathrm{kV-CBCT} \mathrm{exposure} \mathrm{of} 1350 \mathrm{mAs}$.

\subsection{Combining Dose from RT and Imaging}

For radiotherapy, the risk of concomitant imaging needs to be considered in the context of the existing risk of secondary cancer induction from radiotherapy treatment. In addition, the dose to critical organs already receiving high doses from the treatment needs to be assessed to ensure the additional imaging dose does not exceed organ dose limits. The imaging dose needs to be considered both within the treated volume and also peripheral to the volume.

Qiu et al. (2012) performed MC dose calculations for relatively large volume gynecological intensity-modulated RT (IMRT) treatments with field length of $\sim 15 \mathrm{~cm}$, and for CBCT scans of length $\sim 24 \mathrm{~cm}$. They concentrated on modeling the in-field dose, discussing out-of-field dose only briefly. In-field doses for 
organs at risk were calculated using organ equivalent doses calculated using linear, linear-exponential, and plateau radiobiological models. The greatest increment in dose, from imaging one CBCT per fraction, was $2.5 \%$ for the bowel with the linear model but this reduced to $1.3 \%$ for the plateau model. For dose in the peripheral region, the CBCT dose was compared with the linac scatter and leakage doses. In the peripheral low dose regions, where there is low risk of secondary malignancies, the incremental dose from CBCT was found to be an order of magnitude less than the IMRT scatter dose and less than or equal to the linac leakage dose.

Chow et al. (2008) concentrated on in-field dose and compared CBCT dose with the treatment dose for a prostate IMRT case. The planning target volume (PTV) dose rose by $0.6 \mathrm{~Gy}(0.8 \%)$ for a $78 \mathrm{~Gy} / 39$ fractions treatment, which suggests the CBCT dose was $\sim 1.5 \mathrm{cGy}$ per scan. The femoral heads saw the largest increase in dose of $2.5 \mathrm{cGy}(5 \%)$.

Perks et al. (2008) measured the peripheral dose at the center and on the surface of an anthropomorphic phantom. They measured the dose for a prostate IMRT treatment using MOSFETs and $\mathrm{kV}$-CBCT using TLDs. The dose from the IMRT dropped from the prescription dose of $2 \mathrm{~Gy}$ (per fraction) down to $1 \mathrm{cGy}$ at $16 \mathrm{~cm}$ and $0.4 \mathrm{cGy}$ at $21 \mathrm{~cm}$ distance from the field edge. In comparison, the CBCT dose was 0.5 and $0.2 \mathrm{cGy}$ at the same positions, respectively $(7$ and $12 \mathrm{~cm}$, respectively, from the imaged volume edge). They used an S20 collimator which arguably provides a longer field of view than necessary for prostate IGRT. The nominal dose per scan was $6 \mathrm{cGy}$ which they acknowledge was twice that normally used in their clinic. To put this into context, $6 \mathrm{cGy}$ is four times the United Kingdom's diagnostic reference level for imaging the abdomen/pelvis, and is arguably three to five times higher than necessary for adequate image quality for CBCT image guidance (Sykes, 2010).

Harrison and colleagues published two articles on the subject of combined treatment and imaging doses covering anatomical sites of larynx, breast, and prostate (Harrison et al., 2006, 2007). They compared imaging dose from 2D portal imaging and 3D CT imaging with the treatment dose. While this work was not based on CBCT, the differences between CT and CBCT doses are likely to be minimal so the work provides a good perspective on the relative impact of $\mathrm{kV}$ imaging on the combined treatment and imaging dose. For the prostate, they measured dose using TLDs in the rando phantom. Neutron doses were also calculated for the $15 \mathrm{MV}$ beams. They calculated the dose to multiple organs both in-field and out-of-field for combinations for a 37 fraction two phase prostate treatment with $26 \mathrm{CT}$ images and 4 portal images. The excess relative risk (ERR) was found to be $<0.1$ for most organs with bone surfaces, small intestine, and muscle having ERR $<0.3$. Increases in total dose due to portal imaging of up to $20 \%$ were found for bone marrow and bone surfaces. They employed similar methods for the larynx and breast and concluded that the dose to critical organs increased by $5 \%-20 \%$ with increases of up to $30 \%$ for bone surfaces and bone marrow. They noted that by far the largest component of dose to these organs was from scatter and leakage from the MV beam.

Alaei et al. (2014) used a treatment-planning system to compute the imaging dose for head and neck and pelvic treatments and added the imaging dose to the therapeutic one. They showed that high-dose imaging procedures add an appreciable dose to the therapeutic one received by patients. This could become an issue of concern if an organ at risk is proximal, but outside, the treated volume 
but within the volume irradiated by the imaging beam. They also demonstrated the inverse relationship between imaging dose and BMI.

The studies above computed the imaging dose retrospectively. It is, however, beneficial to do so prospectively and account for imaging dose at the time of treatment planning. To this extent, both Alaei et al. (2014) and Grelewicz and Wiersma (2014) combined the $\mathrm{kV}$ imaging and MV therapy beams to perform inverse planning, hence accounting for imaging dose during optimization.

Previous work focused on the dose from kV-CBCT. Combining MV-CBCT imaging dose with the therapeutic one is more straightforward and can easily be accomplished using treatment-planning systems as shown by Miften et al. (2007), Morin et al. (2007a,b), and Akino et al. (2012). Whereas the combination of kVCBCT beams with therapeutic MV ones requires MC codes or other software not commonly available, combining MV-CBCT dose with the therapeutic one can be accomplished routinely in a clinical setting.

One issue with calculating the imaging dose prospectively is that it is not always known what imaging will be required for a particular patient. For instance, an IGRT protocol may have as its basis the use of imaging for a few initial fractions and then weekly thereafter, but if the weekly images show a change in patient setup or anatomy then this may trigger further imaging.

\subsection{Clinical Consequences and Benefits}

\subsubsection{Detrimental Effects of Radiation Exposure}

To date there have been no large-scale epidemiologic studies of the cancer risks associated with $\mathrm{x}$-ray imaging. The evidence we have is derived from measurement and calculation of organ doses and applying organ-specific cancer incidence or mortality data derived from studies of atomic-bomb survivors (Brenner and Hall, 2007). The estimated attributable lifetime risk of death from cancer due to a single, typical, CT scan is $\sim 0.01 \%$ increasing to $0.1 \%$ for exposures in early childhood (Brenner and Hall, 2007). However, these risks are calculated for the general population and not specifically for patients undergoing treatment with radiotherapy. Therefore, the risk of imaging alone is small compared to the risk of treatment failure and other morbidities associated with radiotherapy treatment. It appears sensible, if possible, the risk from the imaging dose should be incorporated into the overall riskcalculation including the treatment dose.

The primary risk of radiation exposure from radiotherapy, including any concomitant imaging but excluding the risks of treatment failure and comorbidities, is the induction of a secondary primary malignancy (SPM). As Tubiana noted in his review (Tubiana, 2009), these rarely occur before 10 years after treatment. However, with increased long-term survival rates, the incidence of these malignancies is likely to increase. Tubiana found from cancer registries that the incidence could be as high as $20 \%$. He also noted that SPMs tended to occur in tissues receiving more than 2 Gy. Data derived from the US Surveillance, Epidemiology, and End Results (SEER) cancer registry by Berrington de Gonzalez et al. (2011) found that $9 \%$ of 5 -year survivors developed a solid tumor and that the relative risk was highest for tissues that typically received more than $5 \mathrm{~Gy}$. From the previous section, we know that the imaging dose is typically small in comparison to the treatment dose. Nevertheless, it adds to the radiation dose burden and contributes to the increased risk of SPM induction. 
Very low doses are also associated with complications. Perks et al. (2008) collected a number of such effects in their paper including prolonged azoospermia at doses $>2.5 \mathrm{~Gy}$ (Howell and Shalet, 1998), loss of ovarian function at doses $<2$ Gy (Wallace et al., 2003), and hypothyroidism or thyroid nodules with median dose equivalents as low as $0.09 \mathrm{~Sv}$ (Imaizumi et al., 2006). Cataract formation can also occur with ERR of $1.98 \mathrm{ERR} / \mathrm{Gy}$ with no lower threshold and with measurable hazard ratios for doses as low as $60 \mathrm{mGy}$ (Chodick et al., 2008).

\subsubsection{Clinical Benefits}

Although there is little doubt of the clinical benefit of CBCT imaging for quality assurance (QA) of patient setup, there is, as yet, little published evidence on improved outcomes attributable to the use of СBCT imaging or other imageguided modalities. Chow et al. (2008) calculated the normal tissue complication probability (NTCP) increase from CBCT imaging during IMRT treatment of the prostate to be $0.5 \%$, although they recognized that the NTCP model used was relatively crude and did not take into account the relative biological effectiveness (RBE) of $\mathrm{kV}$ imaging. Nevertheless, they found that NTCP decreased by $3 \%$ when the clinical target volume (CTV) to PTV margin was reduced from 10 to $5 \mathrm{~mm}$ showing a net benefit of using CBCT imaging for every fraction of treatment. Kron et al. (2010) showed that even when daily online IGRT (CBCT) was used with an adaptive strategy for bladder cancer, the integral dose to both the whole irradiated volume and the irradiated volume minus the CTV was less than the conventional treatment. This is because on average the irradiated volume is smaller in the adaptive strategy than that required otherwise to ensure the bladder is covered the majority of the time. The exception to this was for patients with smaller treatment volumes. Zelefsky et al. (2012) compared cohorts of patients in which one group received prostate IMRT with IGRT, and the other group received the same treatment but without IGRT. They found that biochemical tumor control was significantly better for patients with high-risk prostate carcinoma when IGRT was employed. In addition, late urinary toxicity was almost halved in the group with IGRT. While this study was performed using MV portal imaging and gold seed markers, Moseley et al. (2007) have demonstrated the equivalence of $\mathrm{kV}$-CBCT and gold seed marker versus MV portal image-based IGRT. More recently, Bujold et al. (2012) reviewed the literature and concluded that IGRT has enabled treatments such as hypofractionated stereotactic ablative radiotherapy of the lung, spine, and liver. They also concluded that "an improvement in relapse rate in prostate cancer, Hodgkin disease, and head and neck cancers using IGRT has been consistently reported," and that "there is a suggestion that prostate and head and neck cancer patients might have lower toxicity with IGRT, especially when combined with other technical advances like IMRT.”

\subsection{Closing Remarks}

There are several $\mathrm{x}$-ray imaging options available for IGRT in modern clinical practices. There is a wide variation in the protocols used for these imaging options due to a number of factors. In some cases, requirements for high throughput of patients on machines and availability of suitably trained staff to interpret and act on these images limits the amount of imaging that can be performed. Variable perceptions of risk of imaging dose also contribute to the utilization of x-raybased IGRT. Many centers will use preset image acquisition protocols defined by 
the manufactures. These protocols may well be based on the experiences of early adopters of the equipment; however, there is a lack of evidence and consensus on the minimum image quality required to perform IGRT specific to the particular anatomical site or size of patient. For this reason, the imaging dose may well be higher than necessary. Further work is required in order to optimize image acquisition protocols and the frequency of imaging to achieve the aims of IGRT. To achieve this, standardization of the way radiation dose for imaging is reported needs to be improved.

Further work is also required to understand the risks and benefits attributable to using x-ray-based imaging in RT. The use of dose calculation models to calculate the imaging dose for individual patients may have immediate benefit in estimating the total dose to critical organs/structures of concern, but if implemented for all patients would provide useful data for future analysis. To achieve this, dose calculation algorithms need to be made accessible and integrated efficiently into the clinical workflow so that there are minimal overheads. Ideally, imaging dose calculations would be automated, running in the background and storing the required dosimetric data in the patient record.

\section{References}

AAPM (2009) The role of in-room $\mathrm{kV}$ x-ray imaging for patient setup and target localization. Report of AAPM Task Group 104 of the Therapy Imaging Committee. (One Physics Ellipse, College Park, MD: American Association of Physicists in Medicine).

AAPM (2010) Comprehensive methodology for the evaluation of radiation dose in x-ray computed tomography. Report of AAPM Task Group 111: The future of CT dosimetry. (One Physics Ellipse, College Park, MD: American Association of Physicists in Medicine).

Accuray (n.d.) Estimation of the imaging dose for the CyberKnife ${ }^{\circledast}$ robotic radiosurgerysystem.(http://www.cyberknife.com.tr/images/yayin/Imaging Whitepaper.pdf).

Akino Y et al. (2012) Megavoltage cone beam computed tomography dose and the necessity of reoptimization for imaging dose-integrated intensity-modulated radiotherapy for prostate cancer. Int. J. Radiat. Oncol. Biol. Phys. 82: 1715-1722.

Alaei P, Ding G and Guan H (2010) Inclusion of the dose from kilovoltage cone beam CT in the radiation therapy treatment plans. Med. Phys. 37: 244-248.

Alaei P, Gerbi BJ and Geise RA (2001) Lung dose calculations at kilovoltage X-ray energies using a model-based treatment planning system. Med. Phys. 28: 194-198.

Alaei P and Spezi E (2015) Imaging dose from cone beam computed tomography in radiation therapy Phys. Med. 31: 647-658.

Alaei P, Spezi E and Reynolds M (2014) Dose calculation and treatment plan optimization including imaging dose from kilovoltage cone beam computed tomography. Acta Oncol. 53: 839-844.

Alvarado R, Booth JT, Bromley RM and Gustafsson HB (2013) An investigation of image guidance dose for breast radiotherapy. J. Appl. Clin. Med. Phys. 14(3): 25-38.

Amer A, Marchant T, Sykes J, Czajka J and Moore C (2007) Imaging doses from the Elekta Synergy X-ray cone beam CT system. Br. J. Radiol. 80(954): 476-482. 


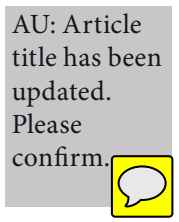

BEIR (2006) Health risks from exposure to low levels of ionizing radiation: BEIR VII Phase 2. (Washington, DC: The National Academies Press).

Berrington de Gonzalez A et al. (2011) Proportion of second cancers attributable to radiotherapy treatment in adults: A cohort study in the US SEER cancer registries. Lancet Oncol. 12(4): 353-360.

Boone JM (2007) The trouble with CTD 100. Med. Phys. 34: 1364-1371.

Borst GR et al. (2010) Clinical results of image-guided deep inspiration breath hold breast irradiation. Int. J. Radiat. Oncol. Biol. Phys. 78: 1345-1351.

Brenner DJ and Hall EJ (2007) Computed tomography-An increasing source of radiation exposure. New England J. Med. 357: 2277-2284.

Bujold A, Craig T, Jaffray D and Dawson LA (2012) Image-guided radiotherapy: Has it influenced patient outcomes? Sem. Radiat. Oncol. 22: 50-61.

Cheng HCY, Wu VWC, Liu ESF and Kwong DLW (2011) Evaluation of radiation dose and image quality for the Varian cone beam computed tomography system. Int. J. Radiat. Oncol. Biol. Phys. 80: 291-300.

Cheung T, Butson MJ and Yu PKN (2003) MOSFET dosimetry in-vivo at superficial and orthovoltage X-ray energies. Australas. Phys. Eng. Sci. Med. 26: 82-84.

Chodick $\mathrm{G}$ et al. (2008) Original contribution risk of cataract after exposure to low doses of ionizing radiation: A 20-year prospective cohort study among US radiologic technologists. Amer J. Epidemiol. 168: 620-631.

Chow JCL (2009) Cone-beam CT dosimetry for the positional variation in isocenter: A Monte Carlo study. Med. Phys. 36: 3512-3520.

Chow JCL et al. (2008) Evaluation of the effect of patient dose from cone beam computed tomography on prostate IMRT using Monte Carlo simulation. Med. Phys. 35: 52-60.

Deng J, Chen Z, Roberts KB and Nath R (2012a) Kilovoltage imaging doses in the radiotherapy of pediatric cancer patients. Int. J. Radiat. Oncol. Biol. Phys. 82: $1680-1688$.

Deng J, Chen Z, Yu JB, Roberts KB, Peschel RE and Nath T (2012b) Testicular doses in image-guided radiotherapy of prostate cancer. Int. J. Radiat. Oncol. Biol. Phys. 82: 39-47.

Ding GX and Coffey CW (2009) Radiation dose from kilovoltage cone beam computed tomography in an image-guided radiotherapy procedure. Int. J. Radiat. Oncol. Biol. Phys. 73: 610-617.

Ding GX and Coffey CW (2010) Beam characteristics and radiation output of a kilovoltage cone-beam CT. Phys. Med. Biol. 55: 5231-5248.

Ding GX, Duggan DM and Coffey CW (2008a) Accurate patient dosimetry of kilovoltage cone-beam CT in radiation therapy. Med. Phys. 35: 1135-1144.

Ding A, Gu J, Trofimov AV and Xu XG (2010) Monte Carlo calculation of imaging doses from diagnostic multidetector CT and kilovoltage cone-beam CT as part of prostate cancer treatment plans. Med. Phys. 37: 6199-6204.

Ding GX and Malcolm AW (2013) An optically stimulated luminescence dosimeter for measuring patient exposure from imaging guidance procedures. Phys. Med. Biol. 58: 5885-5897.

Ding GX and Munro P (2013) Radiation exposure to patients from image guidance procedures and techniques to reduce the imaging dose. Radiother. Oncol. 108: 91-98.

Ding GX, Pawlowski JM and Coffey CW (2008b) A correction-based dose calculation algorithm for kilovoltage x rays. Med. Phys. 35: 5312-5316. 
Dixon RL (2003) A new look at CT dose measurement: Beyond CTDI. Med. Phys. 30: $1272-1280$.

Dixon RL and Boone JM (2010) Cone beam CT dosimetry: A unified and selfconsistent approach including all scan modalities-with or without phantom motion. Med. Phys. 37: 2703-2718.

Downes P, Jarvis R, Radu E, Kawrakow I and Spezi E (2009) Monte Carlo simulation and patient dosimetry for a kilovoltage cone-beam CT unit. Med. Phys. 36: 4156-4167.

Ehringfeld C, Schmid S, Poljanc K, Kirisits C, Aiginger H and Georg D (2005) Application of commercial MOSFET detectors for in vivo dosimetry in the therapeutic X-ray range from $80 \mathrm{kV}$ to $250 \mathrm{kV}$. Phys. Med. Biol. 50: 289-303.

Faddegon BA, Wu V, Pouliot J, Gangadharan B and Bani-Hashemi A (2008) Low dose megavoltage cone beam computed tomography with an unflattened 4 MV beam from a carbon target. Med. Phys. 35: 5777-5786.

Fahrig R, Dixon R, Payne T, Morin RL, Ganguly A and Strobel N (2006) Dose and image quality for a cone-beam C-arm CT system. Med. Phys. 33: 4541-4550.

Falco MD, Fontanarosa D, Miceli R, Carosi A, Santoni R and D'Andrea M (2011) Preliminary studies for a CBCT imaging protocol for offline organ motion analysis: Registration software validation and CTDI measurements. Med. Dos. 36: 91-101.

Flynn RT et al. (2009) Dosimetric characterization and application of an imaging beam line with a carbon electron target for megavoltage cone beam computed tomography. Med. Phys. 36: 2181-2192.

Gayou O, Parda DS, Johnson M and Miften M (2007) Patient dose and image quality from mega-voltage cone beam computed tomography imaging. Med. Phys. 34: 499-506.

Geleijns J, Salvadó Artells M, de Bruin PW, Matter R, Muramatsu Y and McNittGray MF (2009) Computed tomography dose assessment for a $160 \mathrm{~mm}$ wide, 320 detector row, cone beam CT scanner. Phys. Med. Biol. 54: 3141-3159.

Giaddui T, Cui Y, Galvin J, Yu Y and Xiao Y (2013) Comparative dose evaluations between XVI and OBI cone beam CT systems using Gafchromic XRQA2 film and nanoDot optical stimulated luminescence dosimeters. Med. Phys. 40: 062102.

Grelewicz Z and Wiersma RD (2014) Combined MV + kV inverse treatment planning for optimal kV dose incorporation in IGRT. Phys. Med. Biol. 59: 1607-1621.

Gu J, Bednarz B, Xu XG and Jiang SB (2008) Assessment of patient organ doses and effective doses using the VIP-man adult male phantom for selected cone-beam CT imaging procedures during image guided radiation therapy. Radiat. Prot. Dos. 131: 431-443.

Halg RA, Besserer J and Schneider U (2012) Systematic measurements of wholebody imaging dose distributions in image-guided radiation therapy. Med. Phys. 39: 7650-7661.

Harrison RM, Wilkinson M, Rawlings DJ and Moore M (2007) Doses to critical organs following radiotherapy and concomitant imaging of the larynx and breast. Brit. J. Radiol. 80(960): 989-995.

Harrison RM, Wilkinson M, Shemilt A, Rawlings DJ, Moore M and Lecomber AR (2006) Organ doses from prostate radiotherapy and associated concomitant exposures. Brit. J. Radiol. 79 (942): 487-496. 
Hill R, Healy B, Holloway L, Kuncic Z, Thwaites D and Baldock C (2014) Advances in kilovoltage X-ray beam dosimetry. Phys. Med. Biol. 59: R183-R231.

Horakova I and Novak L (2011) Organ and effective doses from verification techniques in image-guided radiotherapy. Radiat. Prot. Dos. 147: 277-280.

Howell S and Shalet S (1998) Gonadal damage from chemotherapy and radiotherapy. Endocrinol Metab Clin North Am. 27(4): 927-943.

$\mathrm{Hu} \mathrm{N}$ and Mclean D (2014) Measurement of radiotherapy CBCT dose in a phantom using different methods. Australas. Phys. Eng. Sci. Med. 37: 779-789.

Hyer DE and Hintenlang DE (2010) Estimation of organ doses from kilovoltage cone-beam CT imaging used during radiotherapy patient position verification. Med. Phys. 37: 4620-4626.

Hyer DE, Serago CF, Kim S, Li JG and Hintenlang DE (2010) An organ and effective dose study of XVI and OBI cone-beam CT systems. J. Appl. Clin. Med. Phys. 11(2): 181-197.

IAEA (2000) Absorbed dose determination in external beam radiotherapy: An international code of practice for dosimetry based on standards of absorbed dose to water. Technical Reports Series No. 398. (Vienna, Austria: International Atomic Energy Agency).

IAEA (2011) Status of computed tomography dosimetry for wide cone beam scanners. Human Health Reports No. 5. (Vienna, Austria: International Atomic Energy Agency).

ICRP (2007) The 2007 recommendations of the International Commission on Radiation Protection. Publication 103. Annals of ICRP 37(2-4).

IEC (2009) IEC 60601-2-44 Medical Electrical Equipment-Part 2-44: Particular requirements for the basic safety and essential performance of X-ray equipment for computed tomography (Third Edition-2009). (Geneva, Switzerland: International Electrotechnical Commission). (https://webstore. iec.ch/publication/2661\&preview=1).

Imaizumi M et al. (2006) Radiation dose-response relationships for thyroid nodules and autoimmune thyroid diseases in Hiroshima and Nagasaki atomic bomb survivors 55-58 years after radiation exposure. JAMA. 295(9): 1011-1022.

IPEMB (1996) The IPEMB code of practice for the determination of absorbed dose for X-rays below $300 \mathrm{kV}$ generating potential $(0.035 \mathrm{Mm}$ Al-4 Mm Cu HVL; $10-300 \mathrm{kV}$ generating potential). Institution of Physics and Engineering in Medicine and Biology. Phys. Med. Biol. 41: 2605-2625.

Isambert A, Ferreira IH, Bossi A, Beaudré A, Nicula LE and Lefkopoulos D (2009) Dose délivrée au patient lors de l'acquisition d'images par tomographie conique de haute énergie. Cancer/Radiother. 13: 358-364.

Islam MK et al. (2006) Patient dose from kilovoltage cone beam computed tomography imaging in radiation therapy. Med. Phys. 33: 1573-1582.

Jacobi W (1975) The concept of the effective dose-a proposal for the combination of organ doses. Radiat. Env. Biophys. 12: 101-109.

Jaffray DA, Drake DG, Moreau M, Martinez AA and Wong JW (1999) A radiographic and tomographic imaging system integrated into a medical linear accelerator for localization of bone and soft-tissue targets. Int. J. Radiat. Oncol. Biol. Phys. 45: 773-789.

Jaffray DA and Siewerdsen JH (2000) Cone-beam computed tomography with a flat-panel imager: Initial performance characterization. Med. Phys. 27: $1311-1323$. 
Jones DG and Shrimpton PC (1991) Survey of CT Practice in the UK Part 3: Normalised organ doses calculated using Monte Carlo techniques. Document NRPB-R250. (Chilton, UK: National Radiological Protection Board).

Jones DG and Wall BF (1985) Organ doses from medical X-ray examinations calculated using Monte Carlo techniques. Report NRPB-R186. (Chilton, Didcot, UK: National Radiological Protection Board).

Kan MW, Leung LH, Wong W and Lam N (2008) Radiation dose from cone beam computed tomography for image-guided radiation therapy. Int. J. Radiat. Oncol. Biol. Phys. 70: 272-279.

Kawrakow I (2013) The EGSnrc Code System: Monte Carlo simulation of electron and photon transport. NRCC Report PIRS-701. (http://irs.inms.nrc.ca/ software/egsnrc/documentation.html).

Kim S, Song H, Samei E, Yin FF and Yoshizumi TT (2011) Computed tomography dose index and dose length product for cone-beam CT: Monte Carlo simulations of a commercial system. J. Appl. Clin. Med. Phys. 12(2): 84-95.

Kim S, Yoshizumi TT, Frush DP, Toncheva G and Yin FF (2010) Radiation dose from cone beam CT in a pediatric phantom: Risk estimation of cancer incidence. Am. J. Roentgenol. 194: 186-190.

Korreman S et al. (2010) Report on 3D CT-based in-room image guidance systems: A practical and technical review and guide. Radiother. Oncol. 94: 129-144.

Koyama S, Aoyama T, Oda N and Yamauchi-Kawaura C (2010) Radiation dose evaluation in tomosynthesis and C-Arm cone-ceam CT examinations with an anthropomorphic phantom. Med. Phys. 37: 4298-4306.

Kron T et al. (1998) Dose response of various radiation detectors to synchrotron radiation. Phys. Med. Biol. 43: 3235-3259.

Kron T, Wong J, Rolfo A, Pham D, Cramb J and Foroudi F (2010) Adaptive radiotherapy for bladder cancer reduces integral dose despite daily volumetric imaging. Radiother. Oncol. 97: 485-487.

Kudchadker RJ, Chang EL, Bryan F, Maor MH and Famiglietti R (2004) An evaluation of radiation exposure from portal films taken during definitive course of pediatric radiotherapy. Int. J. Radiat. Oncol. Biol. Phys. 59: 1229-1235.

Le Heron JC (1992) Estimation of effective dose to the patient during medical X-ray examinations from measurements of the dose-area product. Phys. Med. Biol. 37: 2117-2126.

Ma CM et al. (2001) AAPM protocol for $40-300 \mathrm{kV}$ x-ray beam dosimetry in radiotherapy and radiobiology. Med. Phys. 28: 868-893.

Marinello G, Mege JP, Besse MC, Kerneur G and Lagrange JL (2009) Radiotherapie des cancers de la prostate: Evaluation in vivo de la dose delivre par tomographie conique de basse energie (kV). Cancer/Radiother. 13: 353-357.

Meeks SL, Harmon JF, Langen KM, Willoughby TR, Wagne TH and Kupelian PA (2005) Performance characterization of megavoltage computed tomography imaging on a helical tomotherapy unit. Med. Phys. 32: 2673-2681.

Miften M, Gayou O, Reitz B, Fuhrer R, Leicher B and Parda DS (2007) IMRT planning and delivery incorporating daily dose from mega-voltage cone-beam computed tomography imaging. Med. Phys. 34: 3760-3767.

Montanari D et al. (2014) Comprehensive evaluations of cone-beam CT dose in image-guided radiation therapy via GPU-based Monte Carlo simulations. Phys. Med. Biol. 59: 1239-1253.

Moore CJ et al. (2014) Developments in and experience of kilovoltage X-ray cone beam image-guided radiotherapy. Brit. J. Radiol. 79 Spec No 1 S66-S78. 
Mori S et al. (2005) Enlarged longitudinal dose profiles in cone-beam CT and the need for modified dosimetry. Med. Phys. 32: 1061-1069.

Morin O et al. (2007a) Dose calculation using megavoltage cone-beam CT. Int. J. Radiat. Oncol. Biol. Phys. 67: 1201-1210.

Morin O et al. (2007b) Patient dose considerations for routine megavoltage conebeam CT imaging. Med. Phys. 34: 1819-1827.

Moseley DJ et al. (2007) Comparison of localization performance with implanted fiducial markers and cone-beam computed tomography for on-line imageguided radiotherapy of the prostate. Int. J. Radiat. Oncol. Biol. Phys. 67: 942-953.

Murphy MJ et al. (2007) The management of imaging dose during image-guided radiotherapy: Report of the AAPM Task Group 75. Med. Phys. 34: 4041-4063.

$\mathrm{Ng}$ JA et al. (2012) Kilovoltage intrafraction monitoring for prostate intensity modulated arc therapy: First clinical results. Int. J. Radiat. Oncol. Biol. Phys. 84: e655-e661.

Nobah et al. (2014) Radiochromic film based dosimetry of image-guidance procedures on different radiotherapy modalities. J. Appl. Clin. Med. Phys. 15(6): 229-239.

Nunn AA, Davis SD, Micka JA and DeWerd LA (2008) LiF:Mg, Ti TLD response as a function of photon energy for moderately filtered X-ray spectra in the range of 20-250 kVp relative to 60Co. Med. Phys. 35: 1859-1869.

Osei EK, Schaly B, Fleck A and Charland P (2009) Dose assessment from an online kilovoltage imaging. J. Radiol. Prot. 29: 37-50.

Palm A, Nilsson E and Herrnsdorf L (2010) Absorbed dose and dose rate using the Varian OBI 1.3 and 1.4 CBCT system. J. Appl. Clin. Med. Phys. 11(1): 229-240.

Pawlowski JM and Ding GX (2014) An algorithm for kilovoltage X-ray dose calculations with applications in $\mathrm{kV}-\mathrm{CBCT}$ scans and $2 \mathrm{D}$ planar projected radiographs. Phys. Med. Biol. 59: 2041-2058.

Perks JR, Lehmann J, Chen AM, Yang CC, Stern RL and Purdy JA (2008) Comparison of peripheral dose from image-guided radiation therapy (IGRT) using $\mathrm{kV}$ cone beam CT to intensity-modulated radiation therapy (IMRT). Radiother. Oncol. 89: 304-310.

Poirier Y, Kouznetsov A, Koger B and Tambasco M (2014) Experimental validation of a kilovoltage X-ray source model for computing imaging dose. Med. Phys. 41: 041915.

Qiu Y, Moiseenko V, Aquino-Parsons C and Duzenli C (2012) Equivalent doses for gynecological patients undergoing IMRT or RapidArc with kilovoltage cone beam CT. Radiother. Oncol. 104: 257-262.

Qiu Y, Popescu LA, Duzenli C and Moiseenko V (2011) Mega-voltage versus kilovoltage cone beam $\mathrm{CT}$ used in image guided radiation therapy: Comparative study of microdosimetric properties. Rad. Prot. Dos. 143: 477-480.

Quinn A, Holloway LC, Cutajar DL, Hardcastle N, Rosenfeld AB and Metcalfe PE (2011) Megavoltage cone beam CT near surface dose measurements: Potential implications for breast radiotherapy. Med. Phys. 38: 6222-6227.

Saw CB et al. (2007) Performance characteristics and quality assurance aspects of kilovoltage cone-beam CT on medical linear accelerator. Med. Dos. 32: 80-85.

Sawyer LJ, Whittle SA, Matthews ES, Starritt HC and Jupp TP (2009) Estimation of organ and effective doses resulting from cone beam CT imaging for radiotherapy treatment planning. Brit. J. Radiol. 82(979): 577-584. 
Schiffner DC et al. (2007) Daily electronic portal imaging of implanted gold seed fiducials in patients undergoing radiotherapy after radical prostatectomy. Int. J. Radiat. Oncol. Biol. Phys. 67: 610-619.

Shah AP et al. (2008) Patient dose from megavoltage computed tomography imaging. Int. J. Radiat. Oncol. Biol. Phys. 70: 1579-1587.

Shah A, Aird E and Shekhdar J (2012) Contribution to normal tissue dose from concomitant radiation for two common $\mathrm{kV}-\mathrm{CBCT}$ systems and one MVCT system used in radiotherapy. Radiother. Oncol. 105: 139-144.

Shirato H, Seppenwoolde Y, Kitamura K, Onimura R and Shimizu S (2004) Intrafractional tumor motion: Lung and liver. Sem. Rad. Oncol. 14: 10-18.

Shope TB, Gagne RM and Johnson GC (1981) A method for describing the doses delivered by transmission X-ray computed tomography. Med. Phys. 8: 488-495.

Smith-Bindman R et al. (2009) Radiation dose associated with common computed tomography examinations and the associated lifetime attributable risk of cancer. Arch. Intern. Med. 169: 2078-2086.

Son K, Cho S, Kim JS, Han Y, Ju SG and Choi DH (2014) Evaluation of radiation dose to organs during kilovoltage cone-beam computed tomography using Monte Carlo simulation. J. Appl. Clin. Med. Phys. 15(2): 295-302.

Song WY et al. (2008) A dose comparison study between $\mathrm{XVI}^{\circledast}$ and $\mathrm{OBI}^{\circledR}$ CBCT systems. Med. Phys. 35: 480-486.

Spezi E, Downes P, Jarvis R, Radu E and Staffurth J (2012) Patient-specific threedimensional concomitant dose from cone beam computed tomography exposure in image-guided radiotherapy. Int. J. Radiat. Oncol. Biol. Phys. 83: 419-426.

Spezi E, Downes P, Radu E and Jarvis R (2009) Monte Carlo simulation of an X-ray volume imaging cone beam CT unit. Med. Phys. 36: 127-136.

Spezi E and Lewis G (2008) An overview of Monte Carlo treatment planning. Rad. Prot. Dos. 131: 123-129.

Spezi E, Volken W, Frei D and Fix MK (2011) A virtual source model for kilovoltage cone beam CT: Source characteristics and model validation. Med. Phys. 38: 5254-5263.

Stock M, Palm A, Altendorfer A, Steiner E and Georg D (2012) IGRT induced dose burden for a variety of imaging protocols at two different anatomical sites. Radiother. Oncol. 102: 355-363.

Sykes JR (2010) PhD thesis: Quantification of geometric uncertainties in image guided radiotherapy. University of Leeds, UK.

Sykes JR et al. (2010) Evaluation report X-ray tomographic image guided radiotherapy systems. (http://nhscep.useconnect.co.uk/CEPProducts/Catalogue. aspx).

Sykes JR, Amer A, Czajka J and Moore CJ (2005) A feasibility study for image guided radiotherapy using low dose, high speed, cone beam X-ray volumetric imaging. Radiother. Oncol. 77: 45-52.

Tien CJ, Lee SW and Dieterich S (2014) Estimated clinical impact of fractionation scheme and tracking method upon imaging dose in CyberKnife robotic radiosurgery. Austin J. Nucl. Med. Radiother. 1: 1-5.

Tubiana M (2009) Can we reduce the incidence of second primary malignancies occurring after radiotherapy? A critical review. Radiother. Oncol. 91: 4-15.

Varian Medical Systems (2012) Dose in CBCT-OBI advanced imaging On-Board Imager $\mathrm{kV}$ imaging systems v1.4 and $\mathrm{v} 1.5$. 
Verhaegen F and Seuntjens J (2003) Monte Carlo modelling of external radiotherapy photon beams. Phys. Med. Biol. 48: R107-R164.

Wallace WHB, Thomson AB and Kelsey TW (2003) The radiosensitivity of the human oocyte. Hum. Reprod. 18: 117-121.

Walter C et al. (2007) Phantom and in-vivo measurements of dose exposure by image-guided radiotherapy (IGRT): MV portal images vs. $\mathrm{kV}$ portal images vs. cone-beam CT. Radiother. Oncol. 85: 418-423.

Walters BRB, Ding GX, Kramer R and Kawrakow I (2009) Skeletal dosimetry in cone beam computed tomography. Med. Phys. 36: 2915-2922.

Wen $\mathrm{N}$ et al. (2007) Dose delivered from Varian's CBCT to patients receiving IMRT for prostate cancer. Phys. Med. Biol. 52: 2267-2276.

Winey B, Zygmanski P and Lyatskaya Y (2009) Evaluation of radiation dose delivered by cone beam CT tomosynthesis employed for setup of external breast irradiation. Med. Phys. 36: 164-173.

Xu XG, Chao TC and Bozkurt A (2000) VIP-Man: An image-based whole-body adult male model constructed from color photographs of the visible human project for multi-particle Monte Carlo calculations. Health Phys. 78: 476-486.

Zelefsky MJ et al. (2012) Improved clinical outcomes with high-dose image guided radiotherapy compared with non-IGRT for the treatment of clinically localized prostate cancer. Int. J. Radiat. Oncol. Biol. Phys. 84: 125-129.

Zhang Y, Yan Y, Nath R, Bao S and Deng J (2012) Personalized assessment of kV cone beam computed tomography doses in image-guided radiotherapy of pediatric cancer patients. Int. J. Radiat. Oncol. Biol. Phys. 83: 1649-1654. 